\title{
A MINIATURISED INTEGRATED QCM-BASED ELECTRONIC NOSE MICROSYSTEM
}

\author{
Thomas Frisk ${ }^{1}$, Lars Eng ${ }^{2}$, Shaohua Guo ${ }^{1}$, Wouter van der Wijngaart ${ }^{1}$ and Göran Stemme ${ }^{1}$ \\ 1) Microsystem Technology Laboratory, School of Electrical Engineering, \\ Royal Institute of Technology (KTH), Stockholm, Sweden \\ ${ }^{2)}$ Biosensor Applications AB, Sundbyberg, Sweden
}

\begin{abstract}
We designed, fabricated and successfully tested an integrated miniaturised Quartz Crystal Microbalance (QCM) based electronic nose microsystem. The microsystem is an assembly of four parts: 1. a micromachined gas-liquid interface with integrated fluid channels and electrical conductors, 2 . an anisotropically conductive double-sided adhesive film, 3. a QCM crystal and 4. a polymer cap with fluid and electrical ports. The choice of the multifunctional materials and the geometric features of the four-component microsystem allow a functional integration of a QCM crystal, electrical contacts, fluidic contacts and a sample interface in a single system with minimal assembly effort, a potential for lowcost manufacturing, and a few orders of magnitude reduction in system size $\left(12 * 12 * 4 \mathrm{~mm}^{3}\right)$ and weight compared to commercially available instruments. The system detected ecstasy in the $100 \mathrm{ng}$ range within 30 seconds.
\end{abstract}

\section{INTRODUCTION}

The detection of drugs, explosives and biological substances has become an important part of security activities at airports, harbours and in public transportation. Also military and customs activities include detection and monitoring of chemicals. Environmental monitoring has become an issue of growing importance. In particular, fast, portable, yet sensitive electronic noses are needed.

We previously reported a measurement setup for narcotics detection [1] in which a micromachined interface for airborne sampleto-liquid adsorption is coupled to a downstream commercial QCM sensor bank.

But, in order to further reduce tubing lengths, number of robots, and number of pumps and in general, machine size, we wanted to substitute pumping with diffusion as the major transport mechanism, enabled through miniaturisation. Integration of sample adsorption site and QCM was the target of this study.

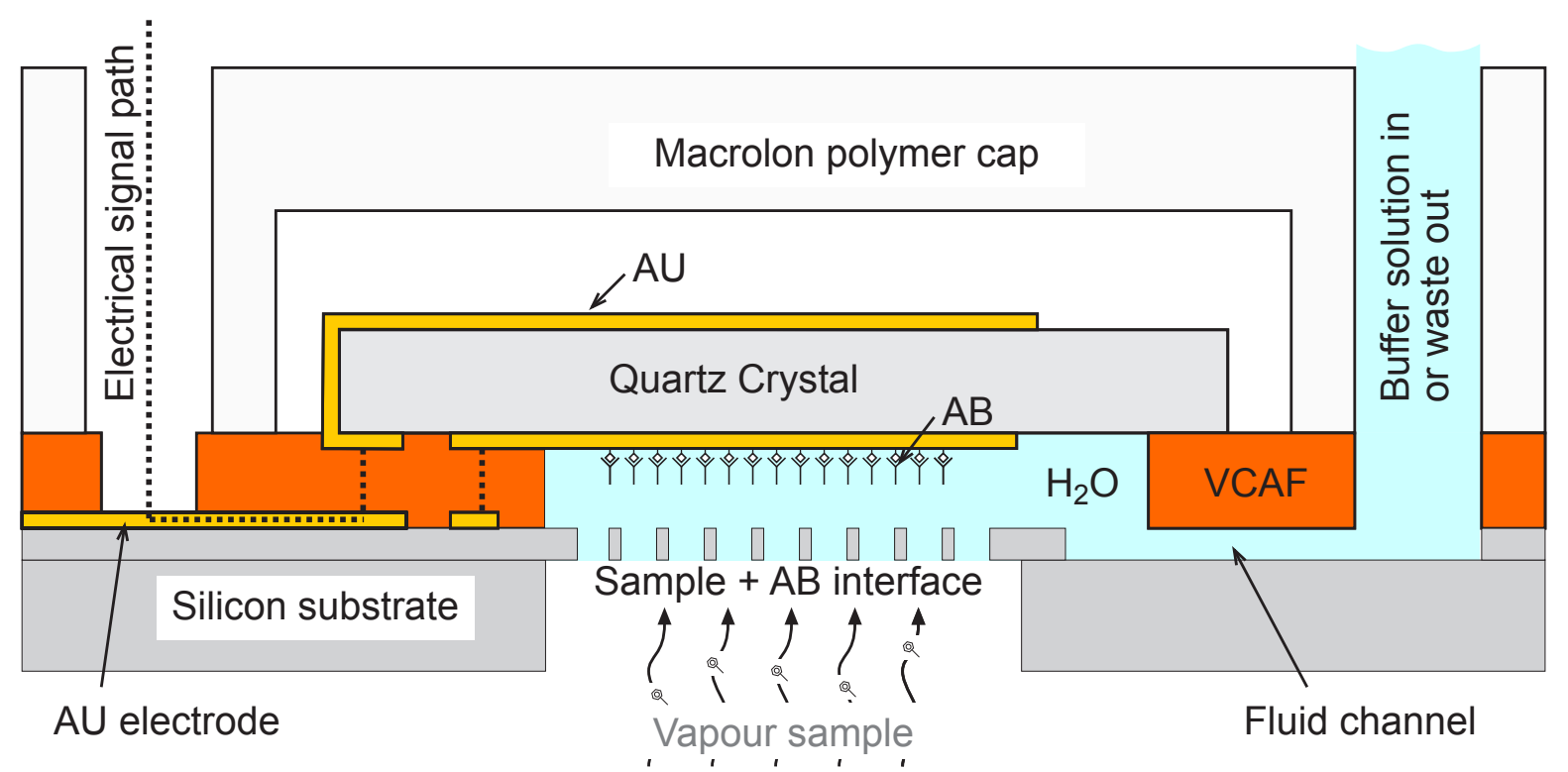

Figure 1. Schematic cross-sectional view of the four-component system. 


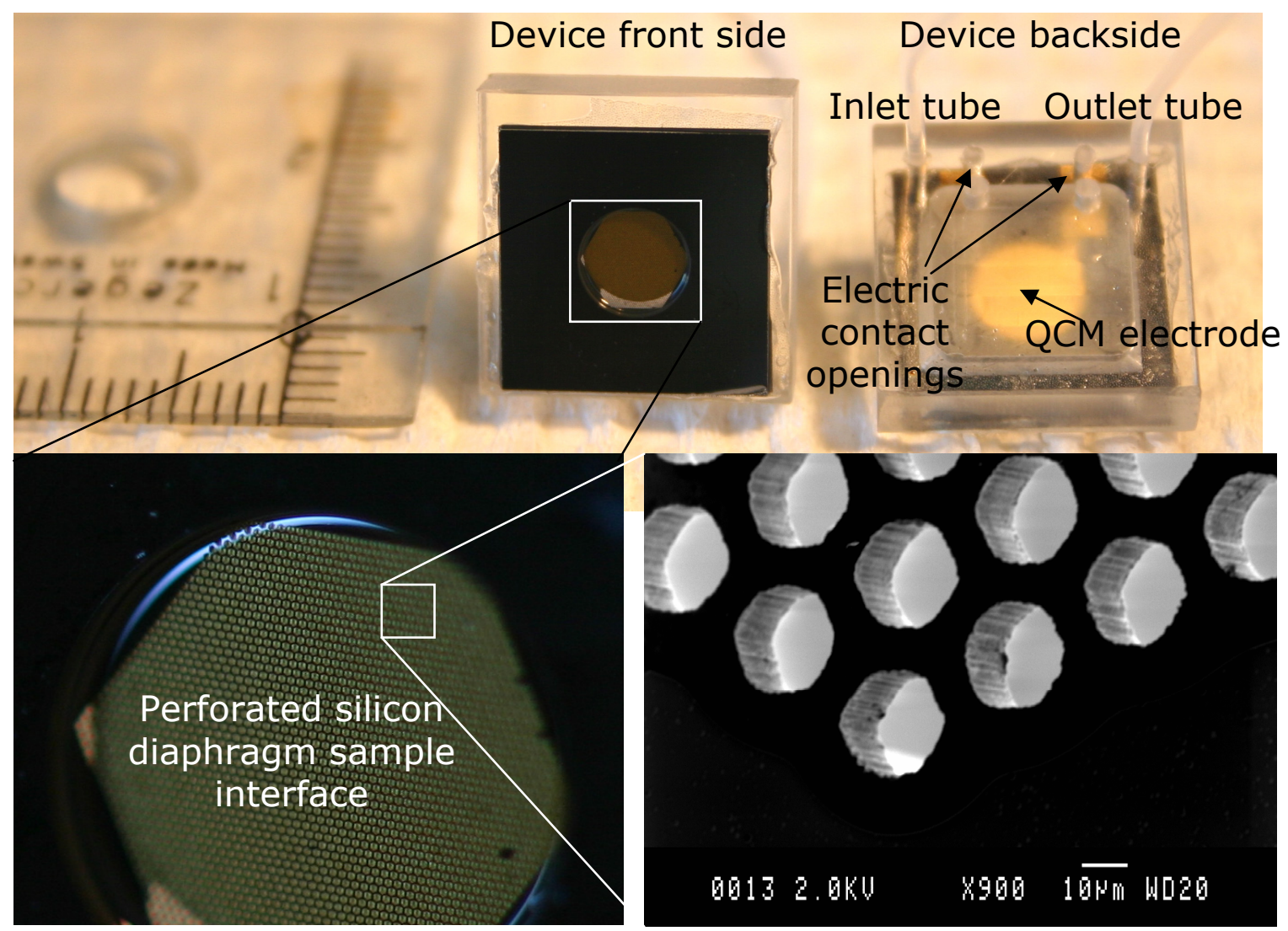

Figure 2. Front and backside picture of two devices. The QCM crystal is visible through the macrolon backside cover cap. The SEM insert shows the $20 \mu \mathrm{m}$ thick diaphragm with hexagon shaped $25 \mu \mathrm{m}$ diameter holes with wall thickness of $10 \mu \mathrm{m}$.

\section{DESIGN}

The current work integrates a novel environment-to-chip sample interface with the QCM sensor element in a single microsystem.

The system consists of four parts. See figures 1, 2 and 3. The first part forms the construction base and consists of a silicon SOI substrate containing electrical contacts, fluidic channels and the interface for airborne sample. The substrate chip was micromachined with standard microfabrication methods including thermal oxidation, gold evaporation, photolithography and deep reactive ion etching (DRIE) of the silicon. Its machined geometry consists of a central $5 \mathrm{~mm}$ diameter, $20 \mu \mathrm{m}$ thick silicon diaphragm with $25 \mu \mathrm{m}$ sized hexagonal perforations.

During operation, liquid surface tension inside the perforations provides a stable, robust and reproducible air-liquid interface. The silicon material provides the required mechanical strength to the diaphragm, which needs to be thin to allow fast sample diffusion, but strong

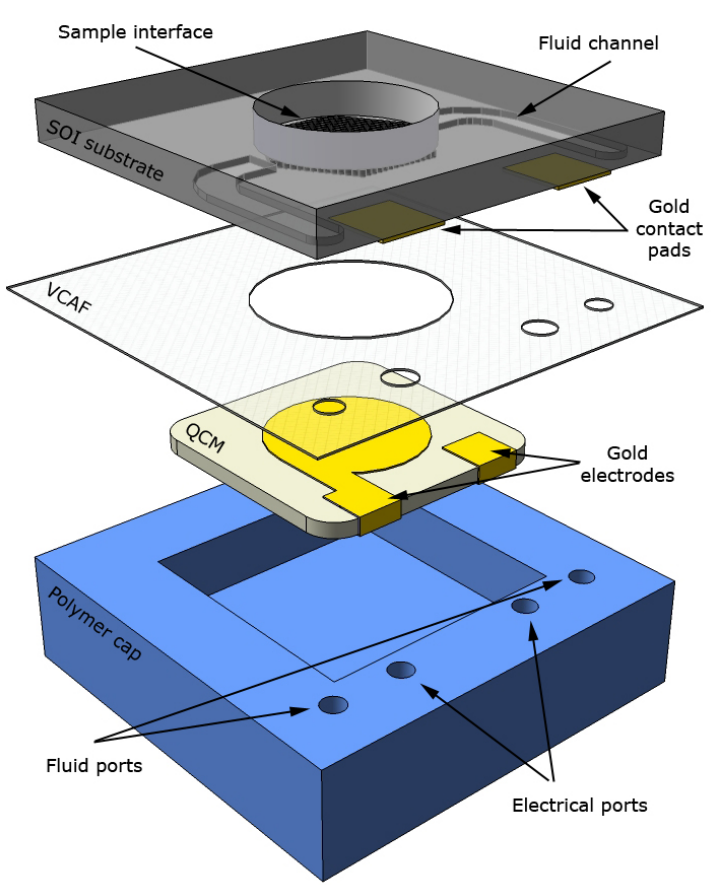

Figure 3. Exploded schematic of the four-component system. 


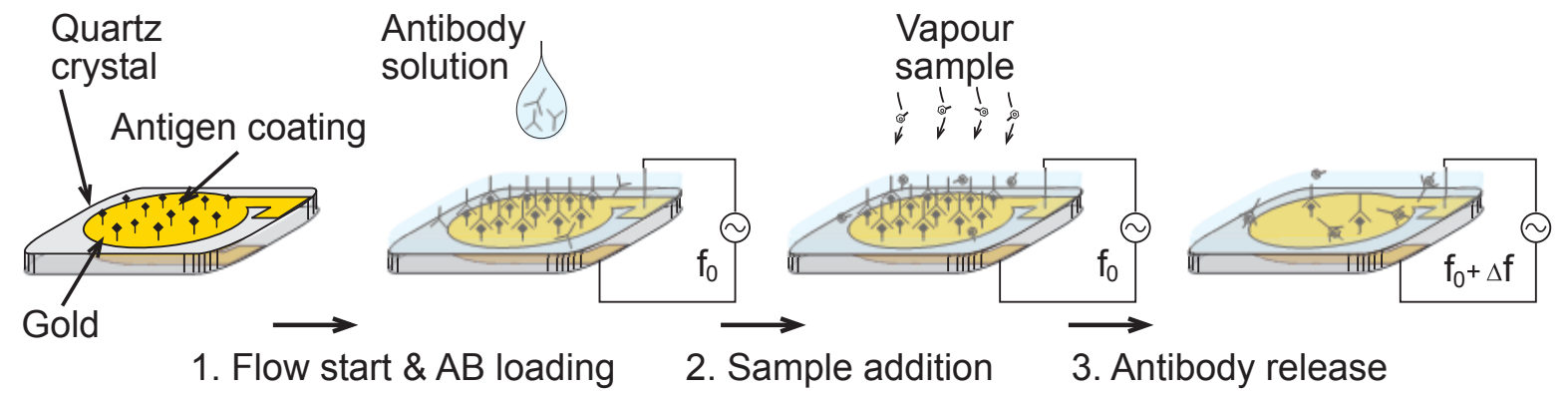

Figure 4. Working principle of a wet QCM during a competitive immunoassay measurement.

enough to withstand the large deformation caused by capillary forces during operation.

The second part of the system consists of a $50 \mu \mathrm{m}$ thick vertically (anistropically) electrically conductive adhesive foil (9703 VCAF; manufacturer: $3 \mathrm{M}$ ). Access ports were punched-out with $5 \mathrm{~mm}$ and $1 \mathrm{~mm}$ punchers and the foil was taped on the silicon substrate surface. To ensure proper bonding between the QCM and the silicon chip, a weight (approximately $100 \mathrm{~g}$ ) was applied during 15 minutes.

The VCAF fulfils four functions:

1. liquid sealing, i.e. restricting the fluids to the appropriate system cavities;

2. mechanical fixing all four parts, including the crystal (!), in a tension-free manner while removing the need for external mechanical clamping;

3. electrical contacting the crystal electrodes to the outside world through anisotropic (z-direction only) conductivity; and

4. avoiding short-circuiting the top and bottom electrode via the xy-direction or through the liquid.

The third part is a commercially available $8 * 8 \mathrm{~mm}^{2}$ QCM with wrap-around gold electrodes. The crystal was pre-coated with antigens and placed on top of the VCAF.

Finally a macrolon polymer cap with openings for fluidic and electrical interfacing was positioned on the VCAF on the silicon chip, covering the crystal without touching it. Tubing (PET $1 \mathrm{~mm}$ ) was attached to the polymer cap for liquid supply of the chemical buffer and for waste removal. Gold pins through the polymer cap touching the contact pads on the chip form the electrical contacts to the oscillator circuit.

Off-microsystem components were limited to a custom made oscillator driver circuit (Biosensor Applications AB, Sundbyberg, Sweden), a computer for frequency readout equipped with DAQ and LabView, a samplefilter with heater for generating airborne narcotics, and a setup consisting of two heightcontrollable recipients for hydrostatic controlled liquid flow through the device.

\section{EXPERIMENTS}

The system was prepared as follows. Phosphate buffer solution (PBS) was continuously transported through the device at $0.35 \mu \mathrm{l} / \mathrm{min}$. This gives a $\sim 3$ minutes residence at the sensing surface.

The antibody mass loading of the QCM was performed by dropping $0.1 \mathrm{~g} / 1$ ecstasy antibody solution onto the air-liquid sample interface (Fig. 4), after which the interface was allowed to return to its fluidic equilibrium state.

The actual measurements were performed as follows. Fibreglass filter sheets containing ecstasy sample were positioned above the interface. They were exposed to an $8 \mathrm{~s}$ heat pulse of $350{ }^{\circ} \mathrm{C}$ to evaporate the sample from the filter. The vaporised airborne sample adsorbs on the exposed liquid surface of the system's interface. The combined effect of diffusion, marangoni flow and convection transports the ecstasy molecules through the $70 \mu \mathrm{m}$ thick liquid layer $(20 \mu \mathrm{m}$ deep perforations plus $50 \mu \mathrm{m}$ liquid layer defined by the VCAF layer thickness) to the QCM surface. Here, the molecules react with the antibodies, removing them from the QCM surface, which causes a resonance frequency increase of the QCM. 


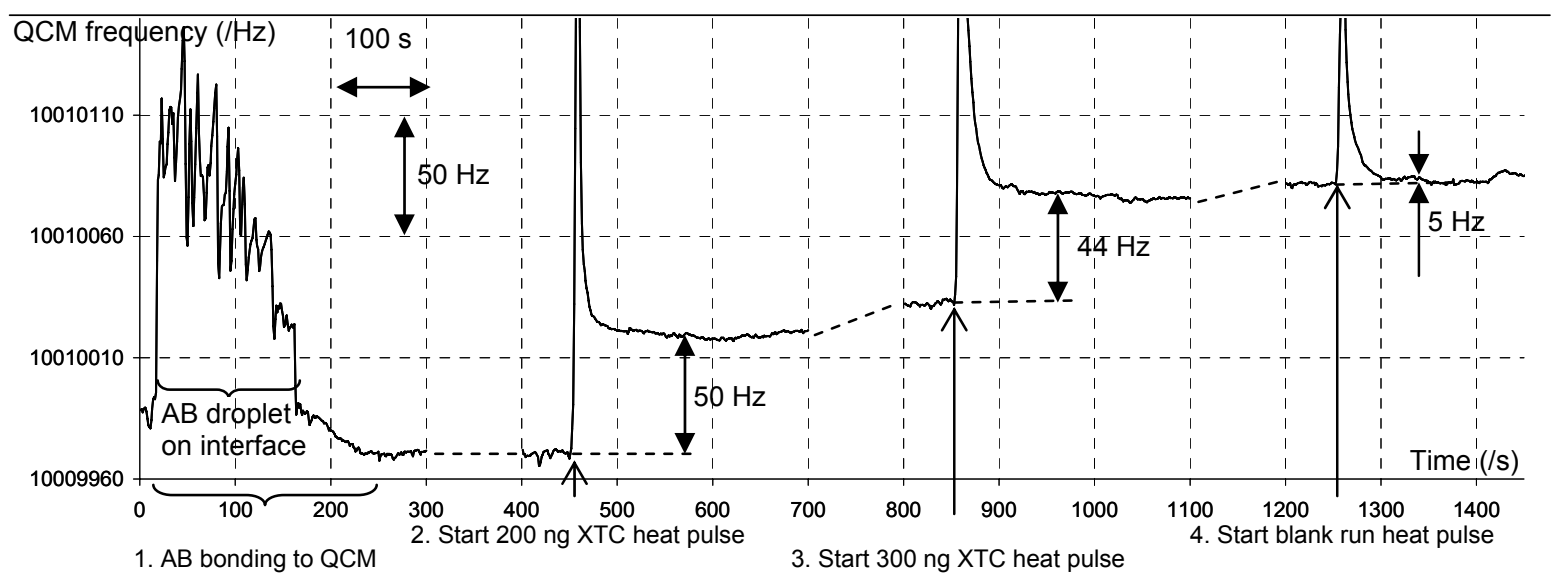

Figure 5. Measured system response to the subsequent loading of the system with 1) antibodies, 2) $200 \mathrm{ng}$ ecstasy vapour, 3) $300 \mathrm{ng}$ ecstasy vapour and 4) a blank run. The extreme pressure sensitivity of the QCM can be observed during the fluidic stabilisation of the interface after droplet addition (step 1), whereas the extreme temperature sensitivity shows in the direct response to the heat pulse (step 4).

\section{RESULTS}

$\mathrm{AB}$ bonding to $\mathrm{QCM}$ resulted in a frequency shift of approximately $25 \mathrm{~Hz}$. After a stabilisation time of 200 seconds the signal was found stable within $\pm 5 \mathrm{~Hz}$.

Consecutive measurements with $200 \mathrm{ng}$ and $300 \mathrm{ng}$ ecstasy (amounts of interest for security applications) on the filter resulted in a respective baseline shift of 50 and $44 \mathrm{~Hz}$ (Fig. 5).

A consecutive final blank filter run resulted in a signal level of at least an order of magnitude lower $(<5 \mathrm{~Hz}$, within noise limits). Also several blank runs with clean filters were performed to evaluate effects of salinity increase caused by evaporation and heat influences on the system, but the baseline shift was found to be within the noise limits.

Similar system tests were also successfully performed with cocaine-cocaine antibody chemistry.

Baseline shift due to changes in ambient conditions (temperature changes caused by handling or people in close proximity to the device) where found to be on a longer time scale than the actual detection time, thus being less of a problem than what we anticipated.

\section{CONCLUSION}

Through using multifunctional materials in combination with micromachined components we constructed an electronic nose microsystem with a minimum of parts count and with a minimum of assembly. We successfully measured $200 \mathrm{ng}$ of narcotics sample with the system. These results are of relevance, both regarding device size, detection levels and detection time.

\section{Corresponding author:}

Thomas Frisk,

Kungliga Tekniska Högskolan - Microsystem Technology Lab, Osquldas väg 10,

SE-10044 Stockholm, Sweden,

Tel: +46-8-7909059, fax: +46-8-100858, email: Thomas.Frisk@ee.kth.se

References: [1] T. Frisk, W. van der Wijngaart, D. Rönnholm and G. Stemme "A Micromachined Interface for Airborne Sample to Liquid Transfer", Lab. Chip., 2006, vol 6, issue 12, 1504-1509. 